\title{
Selenium Nanoparticles-loaded Chitosan Microspheres as a Dietary Selenium Source in Rabbits: Impact on Meat Selenium Content and Oxidative Stability
}

\section{S Fortatos ${ }^{1}$, E Giamouri ${ }^{1}$, AC Pappas ${ }^{1}$, SN Yannopoulos ${ }^{2}$ and G Papadomichelakis ${ }^{1 *}$}

${ }^{1}$ Laboratory of Nutritional Physiology and Feeding, Department of Animal Science, School of Animal Biosciences, Agricultural University of Athens, Greece

${ }^{2}$ Foundation for Research and Technology Hellas - Institute of Chemical Engineering

Sciences (FORTH/ICE-HT), P.O. Box 1414, GR-26504, Rio-Patras, Greece

*Corresponding Author: G Papadomichelakis, Laboratory of Nutritional Physiology and Feeding, Department of Animal Science, School of Animal Biosciences,

Agricultural University of Athens, Greece.
Received: September 20, 2021

Published: October 25, 2021

(C) All rights are reserved by

G Papadomichelakis., et al.

\section{Abstract}

Dietary selenium (Se) supplementation is a viable strategy to enhance the antioxidant defense. The commonly-used Se sources (sodium selenite and selenium-yeast) have a narrow margin between beneficial and toxic effects. Se nanoparticles stabilized in chitosan microspheres (CS-SeNP) are well established for their low toxicity, but their bioavailability and antioxidant potential has not been extensively investigated in livestock feeding. Our objectives were: a) to synthesize and characterize the properties of CS-SeNP and b) to compare the effects of CS-SeNP as dietary selenium source with those of sodium selenite and selenium-yeast on meat selenium concentration and oxidative stability in growing rabbits. The CS-SeNP were synthesized using a chemical reducing method and were characterized by dynamic light scattering, X-ray diffraction and X-ray photoelectron spectroscopy. Four experimental diets were offered to 96 rabbits; one control (C) with no added Se, and 3 diets supplemented with $0.4 \mathrm{mg} \mathrm{Se} / \mathrm{kg}$ either from sodium selenite + selenium-yeast (1:1 ratio; T1), selenium-yeast and CS-SeNPs (1:1 ratio; T2) or CS-SeNP alone (T3). Feed intake, weight gain and feed conversion ratio were monitored throughout the trial. At the end of the trial, 12 rabbits per diet were sacrificed and meat samples were collected. Meat fatty acid composition was determined by gas chromatography. Meat Se content and oxidative stability were determined by hydride (vapor) generation atomic absorption spectroscopy and iron-induced lipid oxidation, respectively. Spherical monodispersed CS-SeNPs of $80.5 \pm 20 \mathrm{~nm}$ average diameter were obtained. The CS-SeNP were exclusively composed of elemental Se and were totally encapsulated in chitosan, as indicated by the X-ray diffraction and X-ray photoelectron spectroscopy surveys, respectively. Growth performance was not affected by the dietary selenium addition and differences were observed between the dietary selenium sources. Meat selenium content and oxidative stability was similar in T1, T2 and T3 rabbits, but significantly higher $(\mathrm{P}<0.05)$ when compared to $\mathrm{C}$ rabbits. In conclusion, the selenium from CS-SeNP enriches meat with selenium and enhances meat oxidative stability in a manner similar to the commonly-used inorganic and organic forms. Given their well-established low toxicity, CS-SeNP have a very good potential as dietary Se source and should be further studied.

Keywords: Meat; Oxidative Stability; Selenium; Selenium Nanoparticles-loaded Chitosan Microspheres; Selenium-yeast; Sodium Selenite 


\section{Abbreviations}

CS: Chitosan; DLS: Dynamic Light Scattering; CS-SeNPs: Selenium Nanoparticles-Loaded Chitosan Microspheres; Se: Selenium; SeNPs: Selenium Nanoparticles; XRD: X-ray Diffraction; XRS: X-ray Photoelectron Spectroscopy

\section{Introduction}

Selenium (Se) is an integral part of at least 25 selenoproteins, some of which are involved in cellular antioxidant defense and redox regulation [1,2]. Animals can readily incorporate dietary Se into edible tissues and produce Se-enriched meat [3] with improved oxidative stability. Indeed, studies in broilers [4] and rabbits [5-7] have showed that feeding supplemental Se may significantly retard oxidation in liver and meat, in addition to tissue enrichment with Se.

To meet the daily requirement of Se and achieve balanced antioxidant status, extra dietary Se supplementation is necessary in animals reared under commercial conditions. The inorganic sodium selenite and organic Se yeast are commonly-used Se sources in the feed industry [8,9], with organic Se being more bioavailable $[10,11]$ and less toxic [12]. Nevertheless, the margin between beneficial and toxic effects in both forms is considered narrow thus, resulting in certain regulations, which limit the maximum addition in all farm animals so that total dietary Se level will not exceed $0.5 \mathrm{mg} / \mathrm{kg}$ [13]. However, there is widespread concern that these recommendations may not be sufficient to prevent selenium deficiency and therefore, there is continued research into alternative selenium sources [14] with greater bioavailability and/or lower toxicity that may be used at higher dietary levels.

With the great progress achieved in the nanotechnology field, selenium nanoparticles (SeNP) have gained considerable attention, because of their unique features such as a high surface activity, high catalytic efficiency, large surface area, strong adsorbing ability, but most importantly low toxicity [15-17]. Acute toxicity tests have indicated that SeNP were much safer than sodium selenite [18] and organic selenium [19], and moreover, exhibited strong antioxidant properties $[18,19]$. However, SeNP usually enlarge, aggregate and finally transform into a gray/black analog that is thermodynamically stable, but biologically inert [18]. Several compounds can be used to stabilize SeNP including but not limited to polysaccharides like chitosan (CS) and proteins such as bovine serum albu- min (BSA) [18]. Generally, BSA as a hydro-soluble protein, could be easily dissolved in the digestive tract of animals thereby releasing SeNPs faster. In contrast, CS might retard the release of SeNP due the deficiency of the enzymes to deal with it in some species of animals and human beings, but is considered superior to BSA in terms of biosafety [18]. In contrast to the expected slower Se release, several studies in mice have showed that selenium nanoparticlesloaded chitosan microspheres (CS-SeNP) is a safe, quite stable form with an acceptable bioavailability and high antioxidant potential $[18,19]$.

The up to date work in rabbits includes limited studies, which mainly used SeNP stabilized with BSA and other factors with quite good effects on semen quality [20] and growth performance [21] in heat-stressed rabbits. However, none of these studies performed a direct comparison of SeNP with the commonly-used inorganic and organic Se forms. Moreover, the potential of SeNP regarding bioavailability and antioxidant potential was not investigated. Therefore, the present study sought to a) synthesize and characterize the physico-chemical properties of CS-SeNP and b) compare the efficacy of CS-SeNP with that of sodium selenite (SS) and Se-yeast (SY) in rabbits, at the recommended by the EU guidelines dietary levels. The comparison between dietary Se forms herein focused mainly on meat Se content and oxidative stability to gain an indirect insight in bioavailability and antioxidant potential.

\section{Materials and Methods}

Synthesis and characterization of selenium nanoparticlesloaded chitosan microspheres

Selenium nanoparticles-loaded chitosan microspheres (CSSeNP) were synthesized by reducing sodium selenite in the presence of chitosan, which adheres to Se atoms and control the size of their aggregation according to the method of Bai., et al. [18] with some modifications. Briefly, $1.0 \mathrm{~g}$ of chitosan (CS, molecular weight 50-190 kDa, 90\% deacylated chitin; Sigma-Aldrich) and $1.6 \mathrm{~g}$ of ascorbic acid were dissolved in $100 \mathrm{ml}$ of $1 \%(\mathrm{w} / \mathrm{w})$ acetic acid. Subsequently, $10 \mathrm{ml}$ of an aqueous solution containing $0.4 \mathrm{~g}$ of sodium selenite was added dropwise into the CS/ascorbic acid solution and stirred continuously (600-800 rpm) to obtain a red SeNP-M colloid. The colloid was dialyzed against $1 \%(\mathrm{w} / \mathrm{w})$ acetic acid (3.5 kDa MWCO dialysis bags) for 6 hours to remove the excess ascorbic acid and other by-products. After that, the colloid was well mixed with a clean CS solution $(3 \mathrm{~g}$ CS in $100 \mathrm{ml}$ of aqueous $1 \%$ 
acetic acid) to achieve final concentrations of $0.09 \%(\mathrm{w} / \mathrm{w})$ Se and $2.0 \%(\mathrm{w} / \mathrm{w}) \mathrm{CS}$. The final colloid was lyophilized and the cake was milled through a 0.5-mm sieve (Cyclotec, 1093 sample mill; Tecator, Höganäs, Sweden) to obtain a bright red free flowing powder, which was stored at room temperature until use.

The hydrodynamic sizes of the CS-SeNP were measured using dynamic light scattering (DLS) on a Mastersizer particle size and zeta potential analyzer (Malvern Instruments, Malvern, UK), with the average size being $80.5 \pm 20 \mathrm{~nm}$. The X-ray diffraction (XRD) was used to study the structure of the CS-SeNP and was performed in the range $5^{\circ} \leq 2 \theta \leq 90^{\circ}$ with the aid of a D8 Advance diffractometer (Bruker Co, Billerica, MA, US), which utilizes the CuKa (1.5406 $\AA$ ) radiation source operated at $40 \mathrm{kV}$ and $40 \mathrm{~mA}$. The X-ray photoelectron spectroscopy (XPS) was used to study the composition of the CS-SeNP and the experiments were carried out in an ultra-high vacuum system using the unmonochromatized AlK $\alpha$ line (1253.6 $\mathrm{eV}$ ). Two analyzer pass energies were used, one at $20 \mathrm{eV}$ (resulting in a full width at half maximum of $0.9 \mathrm{eV}$ for the $\mathrm{Ag} 3 \mathrm{~d} 5 / 2$ peak) and another at $40 \mathrm{eV}$. The XPS core level spectra were analyzed using a fitting routine, which can decompose each spectrum into individual mixed Gaussian-Lorentzian peaks after a Shirley background subtraction. Errors in our quantitative data were found in the range of $\sim 10 \%$, (peak areas) while the accuracy for binding energies (BE) was approximately $0.1 \mathrm{eV}$. The analyzed area on the sample surface was a rectangle with dimensions $7.0 \times 15 \mathrm{~mm}^{2}$.

Animals and diets

Ninety-six healthy 35-day-old weaned New Zealand $\times$ California male animals were purchased from a breeding farm for meat rabbits. Upon arrival at the experimental facilities, they were randomly allocated into four groups, namely control (C), T1, T2 and T3, of 24 rabbits each. They were kept indoors under natural environmental conditions in individual wire mesh cages equipped with metal troughs and automatic nipple drinkers. The rabbits of control group were fed a basal pelleted diet (BD), without any Se supplementation; the only Se present was that of feed ingredients (Table 1). The T1 rabbits were fed the basal diet supplemented with 0.2 $\mathrm{mg} \mathrm{Se} / \mathrm{kg}$ from sodium selenite (SS) and $0.2 \mathrm{mg} \mathrm{Se} / \mathrm{kg}$ from a yeast source (SeY; Sel-Plex®, Alltech Inc, Nicholasville, KY, US). The T2 rabbits were fed the basal diet supplemented with $0.2 \mathrm{mg} \mathrm{Se} / \mathrm{kg}$ from SeY and $0.2 \mathrm{mg} \mathrm{Se} / \mathrm{kg}$ from CS-SeNP, which were synthesized in our laboratory as described above. Finally, the T3 rabbits were fed the basal diet supplemented with $0.4 \mathrm{mg} \mathrm{Se} / \mathrm{kg}$ from CS-SeNP only. These combinations were selected so as to follow the EU recommendations [13] according to which: a) dietary organic Se addition is limited to $0.2 \mathrm{mg} / \mathrm{kg}$, b) the total Se contained in the diet (supplemental and natural occurring) should not exceed $0.5 \mathrm{mg} /$ $\mathrm{kg}$. Thus, the experimental design resulted in the addition of $0.2 \mathrm{mg}$ Se from SS and $0.2 \mathrm{mg}$ Se from SY in T1 and $0.2 \mathrm{mg}$ Se from SY and $0.2 \mathrm{mg}$ Se from CS-SeNP in T2 diets. The T3 diet containing only CS-SeNP was designed for comparison (vs. T1 and T2 diets) reasons. The BD was formulated according to the recommendations for growing rabbits [22]. The ingredients and chemical composition of the diets are shown in table 1.

\section{Experimental procedures}

Handling and care of the experimental animals conformed to the guidelines of the Department of Animal Science. The experimental protocol was approved by the AUA Bioethics Committee (no. 13/16-03-2021). During the experiment, body weight and feed intake were recorded weekly. At the end of the experiment (77 days of age) rabbits were sacrificed by electro stunning and exsanguination, eviscerated and after a $24 \mathrm{~h}$ chilling period at $4{ }^{\circ} \mathrm{C}$, carcasses were weighed and dressing percentage was determined. Subsequently, samples from the Longissimus lumborum (LL) muscle were collected. In detail, from each carcass, the right part of the LL muscle was excised, vacuum packed and stored at $-20^{\circ} \mathrm{C}$ until analyzed for FA. A smaller portion (ca. 2-3 g) from the right part of the LL muscle was used for the determination of Se concentration. Simultaneously, the left part of the muscle was removed, vacuum packed and stored at $-20^{\circ} \mathrm{C}$, so as to determine lipid oxidation.

\section{Determination of selenium in CS-SeNP, diets and meat}

Selenium concentration in feed, CS-SeNP and LL samples was determined using an Agilent 240FS AA atomic absorption spectrometer fitted with VGA77 Vapor generation accessory (Agilent, Santa Clara, USA) based on the method described by Pappas., et al. [23] with minor modifications. In brief, samples ( $0.50 \mathrm{~g})$ were soaked in $4 \mathrm{ml}$ of concentrated HNO3 (65\% w/v, Suprapur; Merck, Germany). The samples were left for 30 minutes for pre-digestion. Then, another $6 \mathrm{ml}$ of concentrated HNO3 were added and then were heated in a microwave accelerated digestion system (CEM, Mars X-Press, Matthews, NC, USA) according to the following program: power was ramped during $20 \mathrm{~min}$ from 100 to $1200 \mathrm{~W}$ and 
Selenium Nanoparticles-loaded Chitosan Microspheres as a Dietary Selenium Source in Rabbits: Impact on Meat Selenium Content and Oxidative Stability

\begin{tabular}{|c|c|c|}
\hline Ingredient & \multicolumn{2}{|c|}{ Basal diet } \\
\hline Dehydrated alfalfa & \multicolumn{2}{|c|}{263.0} \\
\hline Barley grain & \multicolumn{2}{|c|}{266.0} \\
\hline Sunflower meal, 30\% & \multicolumn{2}{|c|}{170.0} \\
\hline Wheat bran & \multicolumn{2}{|c|}{150.0} \\
\hline Sugar beet pulp & \multicolumn{2}{|c|}{120.0} \\
\hline Vegetable oil & \multicolumn{2}{|c|}{14.0} \\
\hline L-Lysine HCl, 80\% & \multicolumn{2}{|c|}{2.5} \\
\hline L-Threonine, 99\% & \multicolumn{2}{|c|}{1.5} \\
\hline DL-Methionine, 99\% & \multicolumn{2}{|c|}{1.0} \\
\hline Calcium carbonate & \multicolumn{2}{|c|}{1.0} \\
\hline Mono-calcium phosphate & \multicolumn{2}{|c|}{1.0} \\
\hline Sodium chloride & \multicolumn{2}{|c|}{2.0} \\
\hline Thyme extract (Addarome) & \multicolumn{2}{|c|}{0.5} \\
\hline Ultrafed $^{\circledR 1}$ & \multicolumn{2}{|c|}{4.0} \\
\hline Premix $^{2}$ & \multicolumn{2}{|c|}{3.5} \\
\hline \multicolumn{3}{|c|}{ Calculated chemical composition } \\
\hline Dry matter & \multicolumn{2}{|c|}{904.0} \\
\hline Organic matter & \multicolumn{2}{|c|}{931.0} \\
\hline Crude protein & \multicolumn{2}{|c|}{157.0} \\
\hline Ether extract & \multicolumn{2}{|c|}{3.4} \\
\hline NDF & \multicolumn{2}{|c|}{338.0} \\
\hline ADF & \multicolumn{2}{|c|}{190.0} \\
\hline Lysine & \multicolumn{2}{|c|}{8.0} \\
\hline Methionine + Cystine & \multicolumn{2}{|c|}{6.0} \\
\hline Threonine & \multicolumn{2}{|c|}{6.7} \\
\hline Calcium & \multicolumn{2}{|c|}{8.0} \\
\hline Phosphorus & \multicolumn{2}{|c|}{5.5} \\
\hline \multirow[t]{2}{*}{ Digestible energy, MJ/kg } & \multicolumn{2}{|c|}{10.2} \\
\hline & & /kg as-fed) \\
\hline Diet & Added $^{3}$ & Determined $^{4}$ \\
\hline $\mathrm{C}$ & - & $0.093 \pm 0.018$ \\
\hline $\mathrm{T} 1$ & 0.40 & $0.508 \pm 0.055$ \\
\hline $\mathrm{T} 2$ & 0.40 & $0.516 \pm 0.060$ \\
\hline $\mathrm{T} 3$ & 0.40 & $0.478 \pm 0.058$ \\
\hline
\end{tabular}

Table 1: Ingredient ( $\mathrm{g} / \mathrm{kg}$ as-fedbasis) and chemical composition ( $\mathrm{g} / \mathrm{kg}$ dry matter) of the basal diet, and selenium (Se) level of the experimental diets.

${ }^{1}$ Contained $>95 \%$ palygorskite $\left[\left(\mathrm{Mg}, \mathrm{Al}_{2} \mathrm{Si}_{4} \mathrm{O}_{10}(\mathrm{OH}) \cdot 4\left(\mathrm{H}_{2} \mathrm{O}\right)\right]\right.$ as agglomerant (binder).

${ }^{2}$ Premix provided per kg diet: vitamin A, 12,000 IU; vitamin D3, 1,200 UI; vitamin E, 50 UI; vitamin K3, 2 mg; vitamin B1, 2.5 mg; vitamin B2, 4 mg; vitamin B6, 2 mg; vitamin B12, 0.02 mg; pantothenic acid, $12.5 \mathrm{mg}$; nicotinic acid, $40 \mathrm{mg}$; folic acid, $1 \mathrm{mg}$; biotine, $0.15 \mathrm{mg}$; choline chloride, 100 mg; I, 1 mg; Mn, 80 mg; Cu, 15 mg; Zn, 80 mg; Fe, 40 mg; Co, 0.5 mg; 300 mg Cycostat (60 mg robenidine/kg). It did not contain any Se source (organic or inorganic).

${ }^{3}$ Se was added as: a) sodium selenite $(0.2 \mathrm{mg} / \mathrm{kg})$ and Se-yeast $(0.2 \mathrm{mg} / \mathrm{kg})$ in T1 diet, b) Se-yeast (0.2 mg/kg) and CS-SeNP (selenium nanoparticles-loaded chitosan microspheres $0.2 \mathrm{mg} / \mathrm{kg})$ in T2 diet and c) CS-SeNP (0.4 mg/kg) in T3 diet; Se-yeast was in the form of SelPlex $^{\circledR}$ (Alltech Inc., Nicholasville, KY, USA); no Se was added to the control (C) diet.

${ }^{4}$ Average of 4 samples per diet \pm standard deviation. 
Selenium Nanoparticles-loaded Chitosan Microspheres as a Dietary Selenium Source in Rabbits: Impact on Meat Selenium Content and Oxidative Stability

held for $15 \mathrm{~min}$. The temperature reached a maximum of $200^{\circ} \mathrm{C}$ followed by a cool-down cycle for $15 \mathrm{~min}$. Losses of volatile element compounds did not occur as the tubes were sealed during heating. The samples were then filtered with disposable syringe filters $0.20 \mu \mathrm{m} / 15 \mathrm{~mm}$ (Chromafil, Macherey-Nagel, Germany). Se ${ }^{\mathrm{VI}}$ reduced to $\mathrm{Se}^{\mathrm{IV}}$ by warming with $6 \mathrm{M}$ concentrated HCL (Merck, Germany) before analysis in the AA atomic absorption system. Calibration standard solutions were prepared from high purity standards. Reductant agent $\mathrm{NaBH}_{4} 0.6 \% \mathrm{w} / \mathrm{v}$ (Sigma- Aldrich, USA) and $\mathrm{NaOH}$ $0.5 \% \mathrm{w} / \mathrm{v}$ (Fisher Scientific, UK) and $10 \mathrm{M} \mathrm{HCl} \mathrm{(Merck,} \mathrm{Germany)}$ was used for vapor generation. To assess the accuracy of the process, two standard reference materials were used, namely RM8414 (Bovine muscle powder, National Research Council, Canada) and RM1577c (Bovine liver, National Institute of Standards and Technology, USA).

\section{Determination of fatty acid profile}

Any external fat and connective tissue were dissected out of the muscle samples, which were then blended in a domestic food processor until smooth. Blending was performed in short bursts to ensure the homogeneous distribution of intramuscular fat in the sample. The FA of diets and intramuscular fat were extracted and methylated directly [24]. Duplicate $1( \pm 0.05)$ g samples were hydrolyzed for $1.5 \mathrm{~h}$ at $55^{\circ} \mathrm{C}$ in $1 \mathrm{~N}$ potassium hydroxide in methanol, containing a known amount (approximately $0.5 \mathrm{mg}$ ) of tridecanoic acid (C13:0) as internal standard. The potassium hydroxide was then neutralized, and the free FAs were methylated by sulphuric acid catalysis $\left(24 \mathrm{~N} \mathrm{H}_{2} \mathrm{SO}_{4}\right)$ for $1.5 \mathrm{~h}$ at $55^{\circ} \mathrm{C}$. Hexane $(3 \mathrm{ml})$ was added to the reaction tube, which was vortex-mixed and centrifuged at $1100 \mathrm{~g}$. The supernatant hexane layer containing the FA methyl esters was kept at $-20^{\circ} \mathrm{C}$, until analyzed by gas chromatography. A temperature-programmed run was followed on a Perkin Elmer Autosystem XL gas chromatograph equipped with a $30 \mathrm{~m} \times 0.25$ $\mathrm{mm} \times 0.25 \mu \mathrm{m}$ internal diameter HP-Innowax capillary column (Agilent Technologies, J\&W GC columns, Santa Clara, CA, USA) and a flame ionization detector (FID). The column temperature was programmed for $1 \mathrm{~min}$ at $140^{\circ} \mathrm{C}$, raised by $2.50 \mathrm{C} / \mathrm{min}$ to $200^{\circ} \mathrm{C}$, then to $230^{\circ} \mathrm{C}$ by $10 \mathrm{C} / \mathrm{min}$ and held for $1 \mathrm{~min}$, and finally to $240^{\circ} \mathrm{C}$ by $4^{\circ} \mathrm{C} /$ min and held for $10 \mathrm{~min}$. Helium was the carrier gas at a constant pressure of 18 psi and the temperature of both the injector and FID was set at $250^{\circ} \mathrm{C}$. Fatty acids were identified by comparison with standards purchased from Sigma-Aldrich Co. (FAME 37 Component; Sigma-Aldrich Co. Supelco, IL, USA) and quantification was achieved using the internal standard (13:0) added prior to hydro- lysis. Total weights of FA (mg/100g) in diets were calculated as the sum of areas for all FA peaks compared to area for $0.5 \mathrm{mg}$ internal standard. Individual FA were expressed as \% by weight of total FA.

\section{Determination of iron-induced lipid oxidation}

Lipid oxidation was determined in raw and cooked meat. The malondialdehyde (MDA) formed during iron-induced lipid oxidation [25,26] was assessed. Briefly, the LL samples were thawed and homogenized in a food processor until smooth. The paste was immediately used to measure lipid oxidation in raw meat. Small patties were formed ( $5 \mathrm{~cm}$ in diameter, $1 \mathrm{~cm}$ thick) and cooked in a domestic microwave oven at $1000 \mathrm{~W}$ for $30 \mathrm{sec}$ until an internal temperature of $70^{\circ} \mathrm{C}$ was reached [27]. Four $1.00( \pm 0.05) \mathrm{g}$ subsamples from the raw or cooked meat were weighed into 50$\mathrm{ml}$ centrifuge tubes and $1.5 \mathrm{ml}$ of a solution containing $1.138 \mathrm{mM}$ ferrous sulphate and $0.368 \mathrm{mM}$ ascorbic acid was added to three of the sub-samples, which were incubated at $37^{\circ} \mathrm{C}$ for either 50 , 150 or $300 \mathrm{~min}$. Following incubation, all three iron-induced subsamples along with the 4th non-induced subsample were immediately homogenized (Polytron homogenizer, PCU, Littau/Lucerne, Switzerland) in the presence of $8 \mathrm{ml}$ of aqueous trichloroacetic acid $(50 \mathrm{~g} / \mathrm{l})$ and $5 \mathrm{ml}$ of butylated hydroxytoluene (BHT) in hexane (8 $\mathrm{g} / \mathrm{l})$, and the mixture was centrifuged. The top layer was discarded and the bottom layer was filtered (Macherey-Nagel no. MN 1640W, Düren, Germany). A 2.5-ml aliquot of the filtrate was mixed with $1.5 \mathrm{ml}$ of aqueous 2 -thiobarbituric acid ( $8 \mathrm{~g} / \mathrm{l})$ and further incubated at $70^{\circ} \mathrm{C}$ for $30 \mathrm{~min}$. Subsequently, the mixture was cooled under tap water and submitted to spectrophotometry (Helios $\alpha$, Thermo spectronic, Cambridge, UK) at $532 \mathrm{~nm}$. The concentration of MDA $(\mathrm{mg} / \mathrm{kg})$ in samples was calculated by referring to slope and intercept data of the standard calibration curve $(0-0.96 \mu \mathrm{M})$ prepared using 1,1,3,3-tetraethoxypropane (TEP), previously hydrolyzed in $0.1 \mathrm{~N} \mathrm{HCl}$.

\section{Statistical analysis}

Data were analyzed using the SPSS statistical package (version 17.0) and are presented as means \pm standard error (SEM). Feed intake, body weight gain, feed conversion ratio, cold carcass weight, dressing percentage and Se content, FA profile in the LL muscle were analyzed by a one-way analysis of variance (ANOVA) with diet as fixed effect. The MDA values were analyzed using a repeated measures ANOVA with diet and time as fixed effects. Post-hoc tests were conducted based on Tukey's criterion. Statistical significance was set at $\mathrm{P}<0.05$ for all tests. 


\section{Results and Discussion}

Characterization of the Se nanoparticles-loaded chitosan microspheres

The DLS analysis showed that monodispersed spherical CSSeNP with an average diameter of $80.5 \pm 20 \mathrm{~nm}$ (mean \pm s.d.) were produced. The XRD pattern showed a totally amorphous structure of the Se in the CS-SeNP, since no traces of Bragg reflections related to crystalline Se were observed (Figure 1). The XPS survey scans revealed the presence of $\mathrm{C}, \mathrm{O}$, and $\mathrm{N}$ atoms on the sample surface (Figure 2a). The $\mathrm{C} 1 \mathrm{~s}$ peak was deconvoluted into three components as shown in figure $2 \mathrm{~b}$. The band located at $284.7 \mathrm{eV}$, could be assigned to C-C or adventitious carbon, which confirmed the presence of the aliphatic group. The band at $286.3 \mathrm{eV}$ was associated with C-N and C-O groups, and the band at $288 \mathrm{eV}$ was attributed to $\mathrm{C}=0$ or $\mathrm{O}-\mathrm{C}-\mathrm{O}$ species. In addition, the N1s peak centered at 399.3 $\mathrm{eV}$ (Figure 2c), could be assigned to $-\mathrm{NH}_{2}$ and $-\mathrm{NH}$ groups. The Se3d peak centered at $55.3 \mathrm{eV}$ (Figure 2d) confirming that the valence state of Se in CS-SeNP was zero $\left(\mathrm{Se}^{0}\right)$. Based on the XPS peak areas, the relative atomic concentration on the surface of CS-SeNP was calculated and presented in table 2. High concentrations of $\mathrm{C}, \mathrm{O}$, and $\mathrm{N}$ atoms (67.97, 28.67 and $3.12 \%$, respectively) corresponding to CS along with traces of Se $(0.25 \%)$ were found on the surface of SeNP-M. The average Se content was determined at $20000 \mathrm{mg} / \mathrm{kg}$ CS-SeNP.

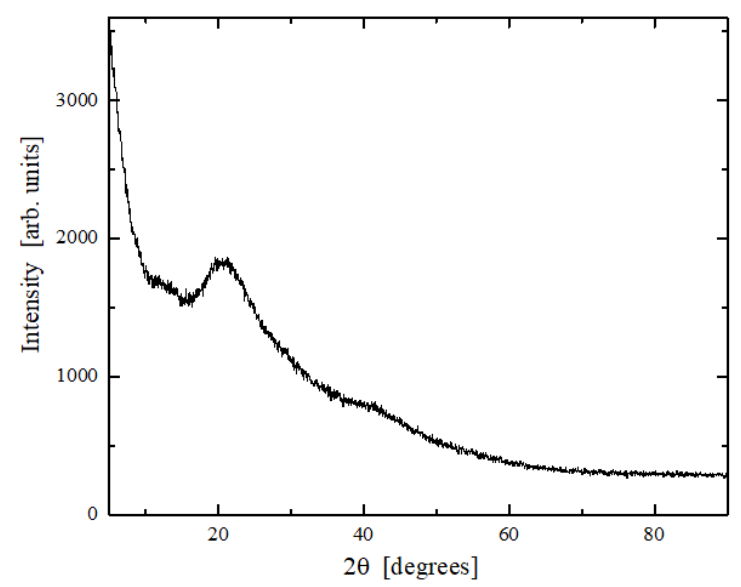

Figure 1: X-ray diffraction (XRD) patterns of the selenium nanoparticles-loaded chitosan microspheres (CS-SeNP).
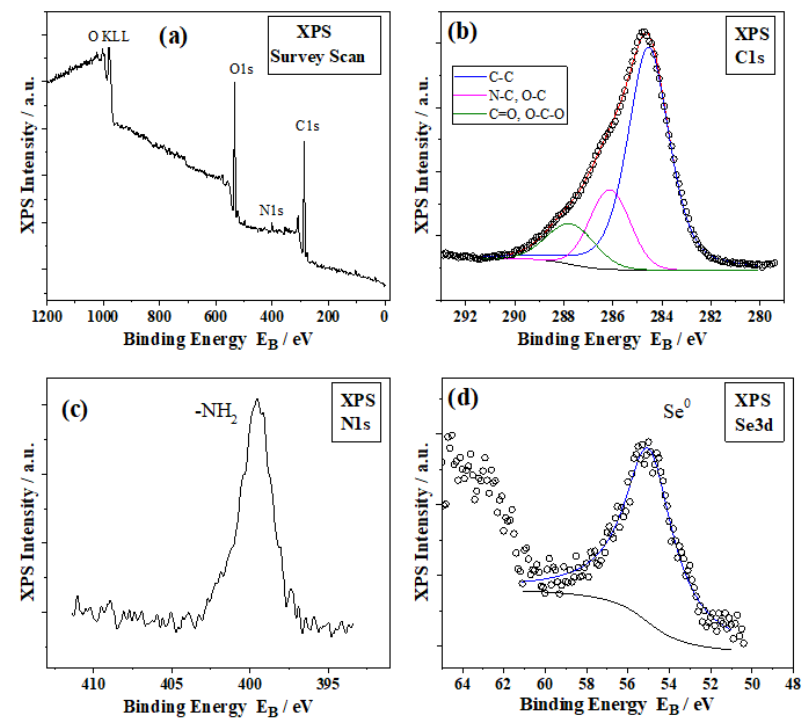

Figure 2: (a) Survey X-ray photoelectron spectroscopy (XPS) spectrum of the selenium nanoparticles-loaded chitosan microspheres (CS-SeNP). (b) Deconvolution of the C1s XPS spectrum into three components. (c) N1s XPS spectrum. (d) Se3d XPS spectrum. Experimental data appear as open circles in (b) and (d), while solid lines are fitting results.

Since CS-SeNP were synthesized in the laboratory using some modifications on a previously published method [18], our first concern was to confirm their characteristics before supplementing the diets. The size distribution analysis conducted using DLS calculated the average diameters of CS-SeNP to be very close ( 80.5 $\pm 20.0 \mathrm{~nm}$ ) to the $95 \mathrm{~nm}$ previously reported [18]. The elemental nature of Se in the CS-SeNP was also confirmed by XPS technique in agreement with Bai., et al. [18]. However, the elemental Se in the CS-SeNP prepared herein was amorphous, in contrast to the crystalline (trigonal phase) feature reported by Bai., et al. [18]. It is not unlikely that differences in the manufacturing conditions between our study and that of Bai., et al. [18] may have affected the nature of Se in the SeNP. Zhang., et al. [28] observed that amorphous elemental Se was present in SeNP encapsulated in high molecular weight CS (200 kDa), whereas crystalline elemental Se was obtained with low molecular weight CS (3 kDa). The crystalline nature of Se when 
Selenium Nanoparticles-loaded Chitosan Microspheres as a Dietary Selenium Source in Rabbits: Impact on Meat Selenium Content and Oxidative Stability

using a very low molecular weight CS (3 $\mathrm{kDa})$ was also reported by Bai., et al. [18]. Herein, we used a moderate to high molecular weight CS (50-190 kDa), which may have resulted in amorphous Se. In general, CS-SeNP with desired properties could be easily prepared in the laboratory and produced in large quantities for the needs of the experiment quite fast. This indicates that CS-SeNP synthesis could possibly be up-scaled to industrial production, as the method adopted here was economic and environment-friendly [18].

\begin{tabular}{|l|c|c|c|}
\hline Element & $\begin{array}{c}\text { Binding } \\
\text { energy (eV) }\end{array}$ & Concentration (\%) & Assignment \\
\hline O1s & 532.53 & $28.67 \pm 0.05$ & C-O \\
\hline C1s & 284.75 & $67.97 \pm 0.06$ & C-C, C-N, O-C \\
\hline N1s & 399.55 & $3.12 \pm 0.04$ & $-\mathrm{NH}_{2},-N H$ \\
\hline Se3d & 55.28 & $0.25 \pm 0.01$ & Se $(0)$ \\
\hline
\end{tabular}

Table 2: Percent relative atomic concentration of elements on the surface of the selenium nanoparticles-loaded chitosan microspheres (CS-SeNP) as determined using the peak areas of the X-ray photoelectron spectroscopy (XPS) spectra.

\section{Growth performance and carcass traits}

The diet did not have any effect on feed intake, growth rate and feed conversion ratio in growing rabbits. Also, no effect was observed on cold carcass weight and dressing percentage (Table 3). Feed intake, weight gain, feed conversion ratio and carcass traits were not affected by the combination of SS with SY (T1 diet), of SY with CS-SeNP (T2) or the CS-SeNP alone (T3) in comparison with the unsupplemented C diet. Abdel-Wareth., et al. [20] reported significantly improved body weight gain in male reproductive rabbits fed diets with nano-elemental Se encapsulated in bovine serum albumin (BSA) due to the improved nutrient digestibility. Sheiha., et al. [21] also observed that rabbits fed with bio- or chemically synthesized Se nanoparticles had better growth performance and dressing percentage when compared to a control diet, with biological nano-Se being more efficient. However, in both studies [20,21] the experiments were carried out under severe heat-stress conditions, which was not the case in the present work; temperature and humidity ranged from $23-26^{\circ} \mathrm{C}$, and $50-70 \%$, respectively. Also, in both studies the Se content in the control diets was not clear and no comparison of nano-Se with inorganic or organic Se forms was con- ducted. Earlier works with dietary inorganic or organic Se in rabbits suggested that positive effects of supplemental Se on growth can be found only when the basal diet is Se-deficient $[5,7]$ or when rabbits are kept under severe environmental conditions [6]. It can be assumed that the Se contained in the basal (control) diet herein satisfied the minimum requirements in growing rabbits [22]; thus, supplemental Se in the form of SS, SY or CS-SeNP was not expected to affect the performance of rabbits.

\begin{tabular}{|c|c|c|c|c|c|c|}
\hline & \multicolumn{4}{|c|}{ Diet $^{1}$} & \multirow{2}{*}{ SEM $^{2}$} & \multirow{2}{*}{$P$-value } \\
\hline & C & T1 & T2 & T3 & & \\
\hline $\begin{array}{l}\text { Initial BW, g } \\
(35 \mathrm{~d})\end{array}$ & 1005 & 1000 & 1013 & 1012 & 34.3 & 0.793 \\
\hline $\begin{array}{l}\text { Final BW, g } \\
(77 \mathrm{~d})\end{array}$ & 2952 & 3010 & 2943 & 2975 & 100.1 & 0.916 \\
\hline ADFI, g/d & 157.9 & 161.2 & 160.6 & 156.3 & 6.48 & 0.863 \\
\hline ADWG, g/d & 47.8 & 49.6 & 47.8 & 48.0 & 1.83 & 0.740 \\
\hline FCR, g/g & 3.31 & 3.27 & 3.35 & 3.26 & 0.071 & 0.537 \\
\hline $\begin{array}{l}\text { Cold carcass } \\
\text { weight, g }\end{array}$ & 1763 & 1813 & 1774 & 1784 & 71.3 & 0.906 \\
\hline $\begin{array}{l}\text { Dressing per- } \\
\text { centage, } \%\end{array}$ & 62.7 & 63.9 & 63.8 & 63.6 & 0.65 & 0.265 \\
\hline
\end{tabular}

Table 3: Effects of diet on body weight (BW), average daily feed intake (ADFI), average daily weight gain (ADWG), feed conversion ratio (FCR) and slaughter traits $(\mathrm{n}=24$ rabbits $/$ diet $)$.

${ }^{1}$ Se source: a) sodium selenite $(0.2 \mathrm{mg} / \mathrm{kg})$ and Se-yeast $(0.2 \mathrm{mg} /$ $\mathrm{kg}$ ) in $\mathrm{T} 1 \mathrm{diet}, \mathrm{b})$ Se-yeast $(0.2 \mathrm{mg} / \mathrm{kg})$ and CS-SeNP (selenium nanoparticles-loaded chitosan microspheres, $0.2 \mathrm{mg} / \mathrm{kg}$ ) in T2 diet and c) CS-SeNP $(0.4 \mathrm{mg} / \mathrm{kg})$ in T3 diet; Se-yeast was in the form of Sel-Plex ${ }^{\circledR}$ (Alltech Inc., Nicholasville, KY, USA); no Se was added to the control (C) diet.

${ }^{2} \mathrm{SEM}=$ Standard error of means.

Meat selenium content, fatty acid composition and oxidative stability

The T1, T2 and T3 diets supplemented with Se increased significantly $(\mathrm{P}<0.05)$ muscle Se content by $196,182 \kappa \alpha$ เ 174\%, respectively, in comparison with the control diet. No differences in the muscle Se content were found between T1, T2 and T3 diets (Figure 3). Based on our experimental design, it is assumed that SS, SY and CS-SeNP enriched meat Se to a similar extent. 


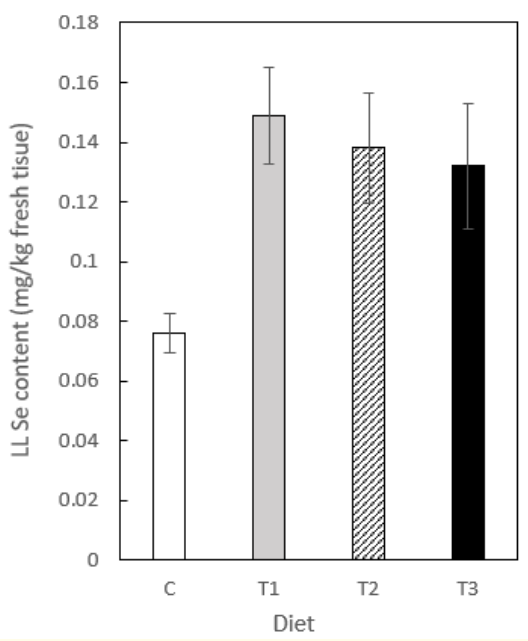

Figure 3: Effect of diet on Se content (mg/kg wet tissue) of Longissimus lumborum (LL) muscle in 77 day-old rabbits ( $\mathrm{n}=12$ rabbits/diet).

One key aspect of dietary Se supplementation in rabbits is enrichment of meat with Se $[6,7,29,30]$ with health promoting effects for humans. The organic forms, such as SY, are considered more effective in increasing muscle Se concentrations [11,30-32], because most Se is in the form of seleno-methionine, which has higher bioavailability [33]. The effect of the dietary nano-Se supplementation on meat Se content and therefore, their bioavailability has not been studied in rabbits. Zhang., et al. [28] reported a Se bioavailability from chemically synthesized CS-SeNP comparable to that of SS in mice. Bai., et al. $[18,34]$ also reported that SeNP in CS-SeNP contributed to the Se retention in a dose-dependent manner in mice, but Se retention of CS-SeNPs was weaker than that of SS. Generally, the SeNP are envisaged widely, particularly in biomedicine, due to their high bioavailability [35]. However, this does not appear to be the case for all SeNP preparations. Our results showed that Se from CS-SeNP accumulated efficiently to muscle tissue, as SS and SY did, despite the fact that CS is considered to delay absorption of Se [18]. Most likely, CS as acid-soluble polysaccharide was dissolved to some extent by the stomach acids or by the free radicals formed by the intestinal cells activity during absorption of nutrients. Such mechanisms have been investigated and proposed in previous studies $[28,34]$.

No major differences in the meat FA composition were detected between diets. Total saturated FA (SFA) were higher $(\mathrm{P}<0.05)$ in T3 compared to T1 and T2 fed rabbits only. The 20:5n-3 was lower $(\mathrm{P}<$ 0.05 ) in T3 compared to T1 fed rabbits only. However, these differences were not enough to substantially modify the polyunsaturated FA (PUFA) or the n-3 content (Table 4) in the intramuscular fat.

\begin{tabular}{|c|c|c|c|c|c|c|}
\hline & \multicolumn{4}{|c|}{ Diet $^{1}$} & \multirow{2}{*}{ SEM } & \multirow{2}{*}{ P-value } \\
\hline & $\mathrm{C}$ & T1 & T2 & T3 & & \\
\hline Total FA & 943 & 932 & 958 & 937 & 30.2 & 0.991 \\
\hline 14:0 & 1.35 & 1.38 & 1.31 & 1.49 & 0.051 & 0.635 \\
\hline 14:1 & 0.11 & 0.17 & 0.17 & 0.12 & 0.013 & 0.234 \\
\hline 15:0 & 0.34 & 0.34 & 0.32 & 0.33 & 0.005 & 0.216 \\
\hline 16:0 & 24.52 & 23.73 & 23.73 & 24.97 & 0.221 & 0.116 \\
\hline $16: 1 n-9$ & $0.33 \mathrm{ab}$ & $0.36 \mathrm{a}$ & $0.32 \mathrm{~b}$ & $0.30 \mathrm{~b}$ & 0.005 & 0.001 \\
\hline 16:1n-7 & 2.46 & 2.97 & 2.92 & 2.55 & 0.167 & 0.628 \\
\hline 17:0 & $0.41 \mathrm{a}$ & 0.39ab & $0.37 \mathrm{~b}$ & $0.37 \mathrm{~b}$ & 0.006 & 0.015 \\
\hline 17:1 & 0.21 & 0.23 & 0.20 & 0.20 & 0.006 & 0.200 \\
\hline 18:0 & $8.35 \mathrm{ab}$ & $8.14 \mathrm{a}$ & $8.63 \mathrm{~b}$ & $8.82 \mathrm{~b}$ & 0.084 & 0.020 \\
\hline $18: 1 n-9$ & 20.74 & 21.05 & 20.61 & 21.26 & 0.279 & 0.851 \\
\hline $18: 1 n-7$ & 1.42 & 1.51 & 1.41 & 1.36 & 0.026 & 0.203 \\
\hline $18: 2 n-6$ & 18.34 & 18.65 & 18.73 & 18.64 & 0.236 & 0.946 \\
\hline
\end{tabular}


Selenium Nanoparticles-loaded Chitosan Microspheres as a Dietary Selenium Source in Rabbits: Impact on Meat Selenium Content and Oxidative Stability

\begin{tabular}{|c|c|c|c|c|c|c|}
\hline $18: 3 n-3$ & 0.94 & 0.93 & 0.92 & 0.97 & 0.020 & 0.775 \\
\hline $20: 1 n-9$ & 0.26 & 0.25 & 0.25 & 0.27 & 0.007 & 0.610 \\
\hline $20: 2$ & 0.78 & 0.81 & 0.80 & 0.73 & 0.016 & 0.315 \\
\hline $20: 3 n-6$ & 0.82 & 0.85 & 0.82 & 0.70 & 0.022 & 0.075 \\
\hline $20: 3 n-3$ & 0.15 & 0.15 & 0.15 & 0.17 & 0.010 & 0.765 \\
\hline $20: 4 n-6$ & 6.01 & 5.84 & 5.95 & 5.38 & 0.179 & 0.605 \\
\hline $20: 5 n-3$ & $0.20^{\mathrm{ab}}$ & $0.22^{\mathrm{a}}$ & $0.20^{\mathrm{ab}}$ & $0.17^{\mathrm{b}}$ & 0.006 & 0.038 \\
\hline $22: 2$ & 0.27 & 0.26 & 0.26 & 0.23 & 0.006 & 0.177 \\
\hline $22: 4 n-6$ & 1.79 & 1.66 & 1.67 & 1.53 & 0.050 & 0.344 \\
\hline $22: 5 n-3$ & 0.75 & 0.74 & 0.72 & 0.64 & 0.025 & 0.400 \\
\hline $22: 6 n-3$ & 0.15 & 0.15 & 0.15 & 0.13 & 0.007 & 0.540 \\
\hline$\Sigma \mathrm{SFA}^{3}$ & $35.16^{\mathrm{ab}}$ & $34.18^{\mathrm{b}}$ & $34.55^{\mathrm{b}}$ & $36.26^{\mathrm{a}}$ & 0.237 & 0.008 \\
\hline${\sum \mathrm{MUFA}^{3}}^{3}$ & 25.88 & 26.88 & 26.25 & 26.45 & 0.463 & 0.901 \\
\hline$\Sigma \mathrm{PUFA}^{3}$ & 30.29 & 30.36 & 30.44 & 29.38 & 0.462 & 0.845 \\
\hline$\Sigma$ PUFA/ $\Sigma$ SFA & 0.87 & 0.90 & 0.89 & 0.82 & 0.018 & 0.423 \\
\hline$\sum \mathrm{n}-3^{4}$ & 2.20 & 2.19 & 2.13 & 2.08 & 0.029 & 0.483 \\
\hline$\sum n-6^{4}$ & 27.05 & 27.10 & 27.25 & 26.33 & 0.430 & 0.885 \\
\hline$\sum \mathrm{LCn}-3^{5}$ & 1.25 & 1.26 & 1.21 & 1.11 & 0.041 & 0.558 \\
\hline
\end{tabular}

Table 4: Effects of diet on total fatty acids (mg/100 g wet tissue) and fatty acid (FA) profile (\% of total FA) of Longissimus lumborum in 77 day-old rabbits ( $\mathrm{n}=12$ rabbits/diet).

1. Se source: a) sodium selenite $(0.2 \mathrm{mg} / \mathrm{kg})$ and Se-yeast $(0.2 \mathrm{mg} / \mathrm{kg})$ in T1 diet, b) Se-yeast (0.2 mg/kg) and CS-SeNP (selenium nanoparticles-loaded chitosan microspheres, $0.2 \mathrm{mg} / \mathrm{kg}$ ) in T2 diet and c) CS-SeNP (0.4 mg/kg) in T3 diet; Se-yeast was in the form of Sel-Plex® (Alltech Inc., Nicholasville, KY, USA); no Se was added to the control (C) diet.

2.SEM= Standard error of means.

3. $\Sigma S F A=$ Sum of saturated fatty acids $(12: 0+14: 0+15: 0+16: 0+17: 0+18: 0), \Sigma M U F A=$ sum of monounsaturated fatty acids $(14: 1+16: 1 \mathrm{n}-$ $7+17: 1+18: 1 n-9+18: 1 n-7+20: 1 n-9), \Sigma P U F A=$ sum of polyunsaturated fatty acids $(18: 2 n-6+18: 3 n-3+18: 3 n-6+20: 3 n-6+20: 3 n-3+20: 4 n-$ $6+20: 5 n-3+22: 4 n-6+22: 5 n-3+22: 6 n-3)$.

4. $\Sigma n-3=$ sum of $n-3$ fatty acids $(18: 3 n-3+20: 5 n-3+22: 5 n-3+22: 6 n-3), \Sigma n-6=$ sum of $n-6$ fatty acids $(18: 2 n-6+18: 3 n-6+20: 3 n-6+20: 4 n-$ $6+22: 4 n-6)$.

${ }^{5} \Sigma \mathrm{LCn}-3=$ sum of long carbon chain $(\geq 20 \mathrm{C}) \mathrm{n}-3$ fatty acids $(20: 3 n-3+20: 5 n-3+22: 5 n-3+22: 6 n-3)$

In a previous study [7], it was reported that dietary supplementation with SY improved muscle FA composition in comparison with an unsupplemented diet, likely through an in vivo protection of polyunsaturated FA or peroxisomal $\beta$-oxidation, which benefits the synthesis of omega-3 (n-3) FA. No such effect was observed herein. This disagreement cannot be fully explained and merits further investigation.
The MDA concentrations in the raw LL samples did not differ between diets at all the designated time points, although a trend for lower MDA concentration was observed in T1, T2 and T3 fed rabbits when compared to control after $300 \mathrm{~min}$ of oxidation. The picture was different in the cooked LL samples, where T1, T2 and T3 fed rabbits showed significantly $(\mathrm{P}<0.05)$ lower MDA concentrations compared to the $\mathrm{C}$ ones after $300 \mathrm{~min}$ of oxidation (Figure 
4). Based again on our experimental design, it is assumed that SS, SY and CS-SeNP had a similar protective role against cooking-induced oxidation.
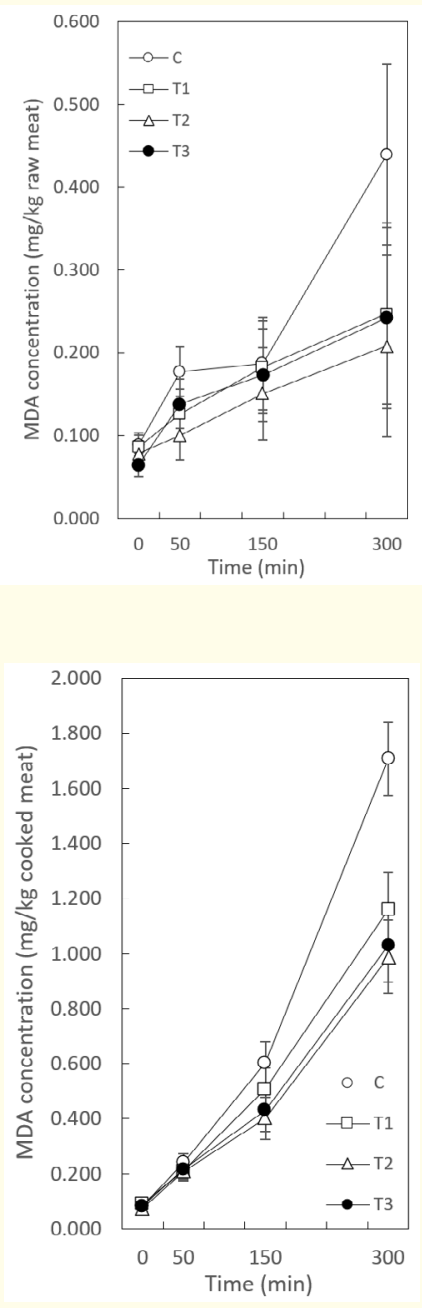

Figure 4: Effect of diet on iron-induced lipid oxidation of Longissimus lumborum in 77 day-old rabbits ( $\mathrm{n}=12$ rabbits/ diet). $\mathrm{MDA}=$ malondialdehyde.

The results indicate that raw meat had a very good oxidative stability, which might be explained by the thyme extract in the basal diet (Table 1). This extract contains essential oils that may have protected the meat from oxidation; therefore, the Se added to diet did not exhibit any significant effects. However, when meat was ad- ditionally stressed by cooking in the microwaves, the protective role of Se (from SS, SY or CS-SeNP) against the control was obvious. Generally, conflicting reports on the effect of dietary Se addition on rabbit meat oxidative stability can be found in literature. In some studies, supplemental SY reduced significantly malondialdehyde (MDA) values during refrigerated storage for 6 days [6] or iron-induced lipid oxidation [7], while no such effect was observed in others $[29,30]$ using SS and/or SY as Se source. The similar oxidative stability of meat in rabbits fed the Se supplemented diets, clearly indicated that CS-SeNP had a significant antioxidant potential, but not superior, as would be expected according to literature, to the commonly-used SS or SY sources. Our results indicate that the elemental Se from CS-SeNP was not only absorbed, it was also biologically active, in accordance Zhang., et al. [36], who was the first to challeng the theory that Se with zero valence state is inactive.

\section{Conclusion}

Meat Se content readily increased and meat oxidative stability was enhanced when CS-SeNP was added in the diet. These changes were similar to those induced by the dietary addition of SS or SY. The present results indicate that CS-SeNP is a source of bioavailable Se and with similar antioxidant potential compared to the commonly used Se sources. The CS-SeNP merits further investigation as feed additive, in terms of alternative (supranutritional) dietary levels.

\section{Acknowledgements}

This Research Project is co-financed by Greece and the European Union (European Social Fund) through the Operational Program "Human Resources Development, Education and Lifelong Learning 2014-2020" and the Program encoded EDBM103, titled "Support for researchers with an emphasis on young researchers-cycle B'”, in the context of the project "Innovative nano-materials in animal nutrition: evaluation of the effects of selenium nanoparticles-loaded chitosan microspheres on meat quality" (MIS 5048474). We are grateful to Bouzas, E. and Danezis, G. for their help during selenium analysis. Dr. L. Sygellou (FORTH/ICE-HT) and Dr. V. Dracopoulos (FORTH/ICE-HT) are thanked for XPS and SEM measurements/ analysis, respectively.

\section{Conflict of Interest}

Declare if any financial interest or any conflict of interest exists. 


\section{Bibliography}

1. Pappas AC., et al. "Selenoproteins and maternal nutrition". Comparative Biochemistry and Physiology 151.4 (2008): 361372.

2. Dalle Zotte A and Szendrő Z. "The role of rabbit meat as functional food". Meat Science 88.3 (2011): 319-331.

3. Surai PF. "Selenium in Nutrition and Health". Nottingham University Press, Nottingham, UK (2006).

4. Pappas AC., et al. "Supranutritional selenium level affects fatty acid composition and oxidative stability of chicken breast muscle tissue". Journal of Animal Physiology and Animal Nutrition 96.3 (2011): 385-394.

5. Zhang Y., et al. "The effect of dietary selenium levels on growth performance, antioxidant capacity and glutathione peroxidase 1 (GSHPx1) mRNA expression in growing meat rabbits". Animal Feed Science and Technology 169.3-4 (2011): 259-264.

6. Ebeid TA., et al. "Fortification of rabbit diets with vitamin $\mathrm{E}$ or selenium affects growth performance, lipid peroxidation, oxidative status and immune response in growing rabbit". Livestock Science 155.2-3 (2013): 323-331.

7. Papadomichelakis G., et al. "Effects of increasing dietary organic selenium levels on meat fatty acid composition and oxidative stability in growing rabbits". Meat Science 131 (2017): 132-138.

8. Yuan D., et al. "Effects of selenium sources and levels on reproductive performance and selenium retention in broiler breeder, egg, developing embryo, and 1-day-old chick". Biological Trace Element Research 144 (2011): 705-714.

9. Surai PF and Fisinin VI. "Selenium in poultry breeder nutrition: an update". Animal Feed Science and Technology 191 (2014): 1-15.

10. Seko $\mathrm{Y}$ and Imura N. "Active oxygen generation as a possible mechanism of selenium toxicity". Biomedical and Environmental Sciences 10.2-3 (1997): 333-339.

11. Payne RL and Southern LL. "Changes in glutathione peroxidase and tissue selenium concentrations of broilers after consuming a diet adequate in selenium". Poultry Science 84.8 (2005): 1268-1276.
12. Fajt Z., et al. "The significance of pork as a source of dietary selenium - an evaluation of the situation in the Czech Republic". Neuro Endocrinology Letters 30.1 (2009): 17-21.

13. EU. "Commission Regulation (EC) No 427/2013 of 8 May 2013 concerning the authorisation of selenomethionine produced by Saccharomyces cerevisiae NCYC R646 as a feed additive for all Animal species and amending Regulations (EC) No 1750/2006, (EC) No 634/2007 and (EC) No 900/2009 as regards the maximum supplementation with selenised Yeast". Official Journal of the European Union L127 (2013): 20-22.

14. Aparna N and Karunakaran R. "Effect of selenium nanoparticles supplementation on oxidation resistance of broiler chicken". Indian Journal of Science and Technology 9.1 (2016).

15. Samak DH., et al. "Developmental toxicity of carbon nanoparticles during embryogenesis in chicken". Environmental Science and Pollution Research International 27.16 (2018): 1905819072.

16. Shahnawaz K., et al. "Impacts of selenium and vitamin E supplementation on mRNA of heat shock proteins, selenoproteins and antioxidants in broilers exposed to high temperature". AMB Express 8.1 (2018): 112.

17. Abdul H., et al. "Green synthesis and characterization of zinc oxide nanoparticles from Ocimum basilicum L. var purpurascens Benth.-lamiaceae leaf extract". Materials Letters 131 (2014): 16-18.

18. Bai K., et al. "Preparation and antioxidant properties of selenium nanoparticles-loaded chitosan microspheres". International Journal of Nanomedicine 12 (2017): 4527-4539.

19. Wang H., et al. "Elemental selenium at nano size possesses lower toxicity without compromising the fundamental effect on selenoenzymes: comparison with selenomethionine in mice". Free Radical Biology and Medicine 42.10 (2007): 15241533.

20. Abdel-Wareth AAA., et al. "Nutritional impact of nano-selenium, garlic oil, and their combination on growth and reproductive performance of male Californian rabbits". Animal Feed Science and Technology 249 (2019): 37-45. 
21. Sheiha AM., et al. "Effects of dietary biological or chemical-synthesized nano-selenium supplementation on growing rabbits exposed to thermal stress". Animals 10.3 (2020): 430.

22. de Blas C and Mateos GG. "Feed formulation". In: de Blas, C., Wiseman, J. (Eds.), The Nutrition of the Rabbit, CAB International, Wallingford, UK (1998): 241-253.

23. Pappas AC., et al. "Effects of supplementing broiler breeder diets with organoselenium compounds and polyunsaturated fatty acids on hatchability". Poultry Science 85.9 (2006): 15841593.

24. O'Fallon JV., et al. "A direct method for fatty acid methylester synthesis: application to wet meat tissues, oils, and feedstuffs". Journal Animal Science 85.6 (2007): 1511-1521.

25. Kornsbrust DJ and Mavis RD. "Relative susceptibility of microsomes from lung, heart, liver, kidney, brain and testes to lipid peroxidation: correction with vitamin E content". Lipids 15 (1980): 315-322.

26. Botsoglou NA., et al. "Effect of dietary oregano essential oil on performance of chickens and on iron-induced lipid oxidation of breast, thigh and abdominal fat tissues". British Poultry Science 43.2 (2002): 223-230.

27. Domínguez R., et al. "Effect of different cooking methods on lipid oxidation and formation of volatile compounds in foal meat". Meat Science 97.2 (2014): 223-230.

28. Zhang C., et al. "Synthesis, characterization, and controlled release of selenium nanoparticles stabilized by chitosan of different molecular weights". Carbohydrate Polymers 134 (2015): 158-166.

29. Dokoupilová A., et al. "Selenium content in tissues and meat quality in rabbits fed selenium yeast". Czech Journal of Animal Science 52.6 (2007): 165-169.

30. Marounek M., et al. "Quality of meat and selenium content in tissues of rabbits fed diets supplemented with sodium selenite, selenized yeast and selenized algae". World Rabbit Science 17.4 (2009): 207-212.

31. Zhan XA., et al. "Effect of different selenium source on selenium distribution, loin quality and antioxidant status in finish- ing pigs". Animal Feed Science and Technology 132.3-4 (2007): 202-211.

32. Pavlata L., et al. "Blood and tissue selenium concentrations in calves treated with inorganic or organic selenium compoundsa comparison". Acta Veterinaria Brno 70.1 (2001): 19-26.

33. Rayman MP. "The use of high-selenium yeast to raise selenium status: how does it measure up?" British Journal of Nutrition 92.4 (2004): 557-573.

34. Bai K., et al. "Selenium-nanoparticles-loaded chitosan/chitooligosaccharide microparticles and their antioxidant potential: a chemical and in vivo investigation". Pharmaceutics 12.1 (2020): 43.

35. Li B., et al. "Biogenic selenium and its hepatoprotective activity". Scientific Reports 7 (2017): 15627.

36. Zhang JS., et al. "Biological effects of a nano red elemental selenium". Biofactors 15.1 (2001): 27-38.

Volume 3 Issue 11 November 2021 (C) All rights are reserved by G Papadomichelakis., et al. 